\title{
Actitudes lingüísticas de profesores mapuche de Educación Básica: vigencia y enseñanza del mapudungun en el contexto educativo
}

\author{
Aldo Olate V.* \\ Marisol Henríquez B. ${ }^{* *}$
}

\begin{abstract}
Resumen
En el presente trabajo se presentarán algunos resultados de un estudio exploratorio y descriptivo que tiene como propósito indagar en las actitudes de profesores de educación general básica frente a la vigencia del mapudungun en contacto con el español. Este estudio se llevó a cabo en dos comunidades mapuche pertenecientes a la VIII Región del Bío Bío y IX Región de la Araucanía. Ambas caracterizadas por su alta densidad poblacional indígena. En esta investigación se seleccionaron tres establecimientos educacionales cuyo proyecto educativo se enmarca dentro del contexto del Programa de Educación Intercultural Bilingüe (PEIB). De estos establecimientos se escogieron seis docentes mapuche a quienes se les aplicó un cuestionario sociolingüístico para relevar las actitudes linguisticas hacia las lenguas en contacto, la vigencia y preservación del vernáculo en la comunidad escolar y las actitudes hacia el PEIB.
\end{abstract}

Palabras Clave: Actitudes lingüísticas - educación intercultural - lenguas en contacto - bilingüismo

\begin{abstract}
This article presents some results of an exploratory and descriptive study wich examines the linguistics attitudes of elementary levels teachers towards the vitality and permanence of the mapudungun language in areas of linguistic contact with spanish. This study was conducted in two mapuche communities from both the VIII region of Bio Bio and the IX region of Araucanía. These regions are characterized by their high density of native population. This research selected three schools in wich the Programa de Educación Intercultural Bilingüe (PEIB) was incorporated as part of their educational projects. Six mapuche teachers of these schools were selected to answer a sociolinguistic questionaire in order to know their linguistics attitudes towards the language in contact, the vitality and permanence of the mapudungun in the educational community and their attitudes toward the PEIB.
\end{abstract}

KeyWords: Linguistics Attitudes- intercultural education - bilingualism - languages in contact.

* Magíster en Lingüística; Dr. @ en Lingüística. Universidad de la Frontera. aolate@ufro.cl

* Magíster en Lingüística; Dra. @ en Lingüística. Universidad Católica de la Santísima Concepción. mhenriquez@ucsc.cl 
Actitudes lingüísticas de profesores mapuche de Educación Básica: vigencia y enseñanza del mapudungun en el contexto educativo / Aldo Olate Vinet y Marisol Henríquez Barahona

\section{Desarrollo argumentativo}

\section{1. Actitudes lingüísticas. Nociones preliminares}

Existe consenso entre los investigadores sobre la importancia que los estudios de las actitudes lingüísticas tienen en el campo de la sociolingüística. Cumplen un rol trascendental en el proceso de difusión de los cambios lingüísticos, en la definición de comunidades de habla, en la consolidación de los patrones de uso y de evaluación social, y, en general, en una amplia gama de fenómenos estrechamente relacionados con la variación lingüística en la sociedad (López Morales, 1993; Fasold, 1996; Moreno Fernández, 2005).

López Morales (1993) destaca el papel decisivo que cumplen las actitudes en la explicación de la competencia comunicativa de los hablantes, la influencia que tienen en el aprendizaje de segundas lenguas y, en el hecho de que pueden fomentar la discriminación lingüística.

Las actitudes lingüísticas son el reflejo de disposiciones o modos, relacionados con la lengua, que tienen miembros de grupos étnicos diferentes y, de acuerdo con Fasold (1996), existen pruebas de que éstas pueden influir en la manera en que los profesores tratan a los alumnos. Asimismo, los esfuerzos que se realizan en diversos países por planificar la conducta lingüística de un pueblo pueden evaluarse a través del estudio de las actitudes lingüísticas de los sujetos hacia las medidas adoptadas por los organismos gubernamentales y educacionales, Silva Corvalán (2001).

Desde la perspectiva de la etnografía de la comunicaciòn, se conciben las actitudes lingüísticas, distinguiéndolas de las actitudes en general, por su referencia explícita hacia la lengua. Saville Troike (2005) sostiene que una de las razones por las cuales las actitudes son particularmente interesantes, se vincula con la idea de que los individuos raramente están en libertad para elegir qué actitudes tener hacia una lengua o variedad. Lo anterior, conlleva a la idea de que éstas se adquieren como un factor de pertenencia de grupo, en este sentido, forman parte del proceso de enculturación que ocurre en una comunidad de habla particular. Las actitudes, siguiendo la reflexión de 
la autora, están determinadas por la cultura e influenciadas por la estructura social de la comunidad.

De este modo, por ejemplo, una actitud favorable o positiva influye en la elección o uso de una lengua en detrimento de otra, ayuda a que un cambio lingüístico se cumpla más rápidamente o que la enseñanza-aprendizaje de una lengua extranjera sea más eficaz. Así también propicia el que algunas variedades se confinen a los contextos menos formales y otras predominen en los estilos cuidados. Por otro lado, una actitud negativa o desfavorable puede llevar al abandono y olvido de una lengua o impedir la difusión de una variante o cambio lingüístico, Moreno Fernández (2005). Las actitudes lingüísticas se manifiestan no sólo hacia las variedades y los usos lingüísticos propios sino también hacia los ajenos. De este modo, a la hora de formarse esa actitud, suelen ser factores decisivos el nivel de estandarización de la lengua y su vitalidad.

\subsection{Enfoque adoptado}

El concepto de actitud al cual nos adscribimos se define en torno a las reacciones y juicios de valor que sostienen los hablantes. De esta forma, las actitudes, en cuanto reacciones, y las creencias, en tanto juicios de valor, se perfilan como valoraciones positivas o negativas que tienen los hablantes hacia una lengua o variedad determinada.

Las actitudes adquieren un valor adicional en relación al campo de estudio sociolingüístico, toda vez que se indague dicho fenómeno en situaciones de convergencia/contacto lingüística/o, porque es dentro de este contexto donde ocurren casos en los que se observan asimetrías sociolingüísticas sistemáticas entre las lenguas. En otras palabras, el estudio de las actitudes toma valor allí donde existen minorías étnicas y lingüísticas. Dentro de este planteo general, la actitud hacia la lengua puede interpretarse como un factor significativo en cuanto a la convivencia, vitalidad y utilidad de las variedades de lenguas en un territorio geográficamente delimitado. Así, García Marcos (1993) sostiene que las actitudes hacia la lengua, al margen del análisis variacionista, pueden influir en los procesos de conservación y desplazamiento de dos o más lenguas. En esta misma línea, éstas pueden determinar los dominios de uso y funciones comunicativas de los códigos, así como también los procesos de adquisición y aprendizaje de una lengua.

\subsection{Educación Intercultural Bilingüe en Chile. Antecedentes generales}

En Chile, la Educación Intercultural Bilingüe es una experiencia de inserción reciente en el sistema educativo nacional, ya que fue instaurada en el Ministerio de Educación en 1996, con base a la promulgación de la ley No 19.253 de 1993, la cual, en el título IV, párrafo 1, artículo 28 contempla el "Reconocimiento, 
Actitudes lingüísticas de profesores mapuche de Educación Básica: vigencia y enseñanza del mapudungun en el contexto educativo / Aldo Olate Vinet y Marisol Henríquez Barahona

Respeto y Promoción de las Culturas Indígenas" y, entre otras acciones, destaca el uso y conservación de los idiomas indígenas, junto al español en las áreas de alta densidad indígena. Para cumplir con lo estipulado por la Ley, la Corporación Nacional de Desarrollo Indígena (CONADI) en coordinación con el Ministerio de Educación (MINEDUC) promovió planes y programas de fomento de las culturas indígenas. De este modo, a partir del año 1996 se acuerda desarrollar proyectos pilotos en escuelas y liceos, cuyas matrículas tengan un alto porcentaje de población indígena. Así, se crea el Programa de Educación Intercultural Bilingüe (PEIB) en el Ministerio de Educación. Programa que se propone buscar la pertinencia de los procesos educativos impulsados por la reforma educacional en marcha (www.mineduc.cl).

El objetivo general de este Programa consiste en "Contribuir a mejorar los logros de aprendizaje, a partir del fortalecimiento de la identidad étnica de las niñas y los niños de establecimientos educacionales de Educación Básica ubicados en contextos de diversidad cultural y lingüística" (www.mineduc.cl).

La concepción educativa que se sostiene, consiste "en un diálogo multidireccional que aspira a educar a los sujetos en la pedagogía de la diversidad. Prepara a los individuos para relacionarse con mundos distintos al propio en igualdad de oportunidades y capacidades, impulsando desde el espacio educativo, la defensa del principio de la pluriculturalidad" (www.mineduc.cl).

En resumen, para el MINEDUC, este Programa se justifica no sólo por los bajos niveles de escolaridad de la población indígena, el bajo rendimiento y logros de aprendizajes de los escolares indígenas en relación a las metas del sistema, sino que también porque los conocimientos y saberes de los pueblos indígenas, se constituyen en una oportunidad para avanzar en la construcción de una sociedad que se enriquezca en la diversidad.

\subsection{El docente como agente activo en la difusión de las actitudes.}

El rol que juega el profesor en la dimensión actitudinal es fundamental, toda vez que es el encargado de la formación del alumnado a lo largo de toda la etapa escolar formal. Desde esta perspectiva, el profesor juega un papel clave en el proceso de enculturación o asimilación que viven los alumnos.

La etnografía de la educación (Fasold, 1998), disciplina que estudia los conflictos culturales entre las escuelas y los grupos minoritarios, destaca la función primaria del docente como agente difusor de valores y actitudes que son propios de la sociedad dominante. En este sentido, los docentes se constituyen como agentes activos en cuanto al fortalecimiento y/o debilitamiento de la vitalidad cultural y lingüística de una minoría étnica. 


\section{La investigación}

\subsection{Propósito}

El presente trabajo tiene como objetivo general analizar algunas actitudes lingüísticas, con respecto a la lengua mapuche y la española, que tienen docentes que se desempeñan en escuelas insertas en Programas de Educación Intercultural Bilingüe en zonas de alta densidad indígena de la VIII y IX Regiones de Chile. La situación sociolingüística general de las poblaciones mapuche pertenecientes a estas regiones se establecen a partir de los trabajos realizados por Gundermann et al. (2008, 2009) Vergara y Gunderman (2005).

Específicamente, analizaremos las actitudes lingüísticas de los docentes en torno a tres parámetros de análisis: vitalidad, lealtad y utilidad de ambas lenguas.

Además, abordaremos, en términos generales, la evaluación que hacen los docentes del Programa de Educación Intercultural Bilingüe de sus escuelas.

\subsection{Metodología}

\subsubsection{Tipo de investigación}

El estudio realizado tuvo carácter exploratorio. El medio para recoger la información fue la aplicación de un cuestionario. El análisis se basó en un enfoque cualitativo y cuantitativo. Dado el carácter exploratorio del estudio no se empleó otra técnica de recogida de información para efectuar el proceso de interpretación y cuantificación de los datos.

\subsubsection{Participantes}

Los participantes fueron 6 profesores en ejercicio en el primer y segundo ciclo de enseñanza básica ( $1^{\circ}$ a $8^{\circ}$ año básico) pertenecientes a 3 escuelas rurales, que están o han estado insertas en el Programa de Educación Intercultural Bilingüe (PEIB). Los establecimientos seleccionados pertenecen a las Provincias de Arauco y Cautín de la VIII y IX Regiones respectivamente. Las zonas rurales en las que están situadas las escuelas se caracterizan por sus altos índices de población mapuche.

\subsubsection{Recolección del material sociolingüístico}

Para la recolección de los datos se diseñó un cuestionario sociolingüístico compuesto por preguntas cerradas y abiertas, el cual se aplicó a los profesores colaboradores y que trató sobre el uso y vigencia del mapudungun en contacto con el español en la escuela y en la comunidad. Dicho instrumento contempló, además, preguntas sobre implementación y efectividad del PEIB.

$$
107
$$


Actitudes lingüísticas de profesores mapuche de Educación Básica: vigencia y enseñanza del mapudungun en el contexto educativo / Aldo Olate Vinet y Marisol Henríquez Barahona

\section{Análisis y discusión los resultados}

\subsection{Uso y frecuencia de uso en la comunidad}

De acuerdo con lo declarado por los docentes, datos presentados en el gráfico 1 , el mapudungun es una lengua hablada fundamentalmente por los ancianos y ancianas (45\%) y también por los adultos en un porcentaje menor (30\%). Dato que corrobora la tendencia presentada por Croese (1980). Sin embargo, el grupo de los niños y niñas es el que tiene menor competencia del vernáculo, siendo el castellano la lengua de uso mayoritario entre los escolares. Todos hablan castellano, pero sólo algunos (9\%) poseen algún grado de desempeño en lengua vernácula.

Gráficol. Quienes hablan mapuche en la comunidad y con qué frecuencia

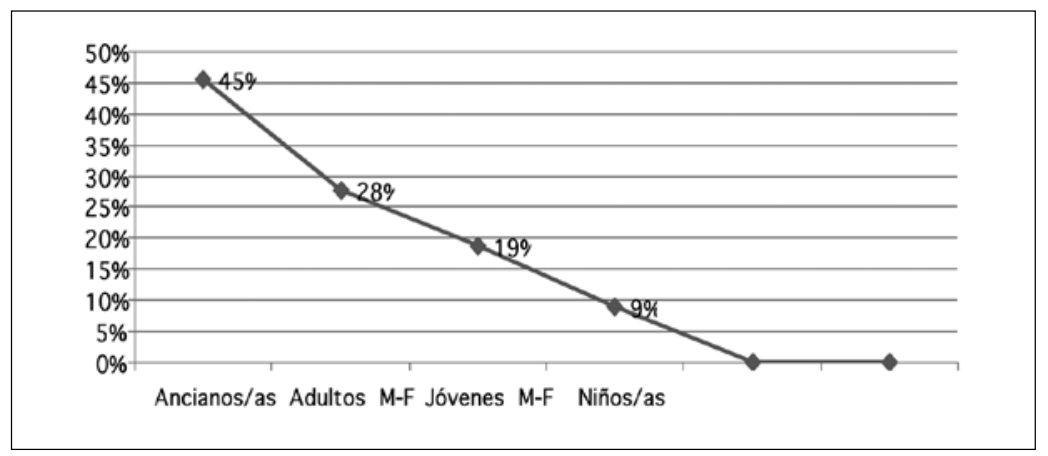

\subsection{Uso y frecuencia de uso en la escuela}

Como se muestra en el gráfico 2, el vernáculo se habla en la escuela, pero "pocas veces" (53,3\%). Estos datos deben considerarse tomando en cuenta dos hechos, por una parte la difusión del mapudungun en las escuelas hasta hace unas décadas era prohibida y castigada, por otro lado, la lengua se ha ido introduciendo poco a poco en la escuela, creemos que a raíz de la implementación de los Programas de Educación Intercultural Bilingüe. La lengua mapuche se habla en la casa, en las ceremonias religiosas y en un \% menor se está comenzando a hablar en la escuela. 
Gráfico 2. Quiénes hablan mapuche en la escuela y con qué frecuencia.

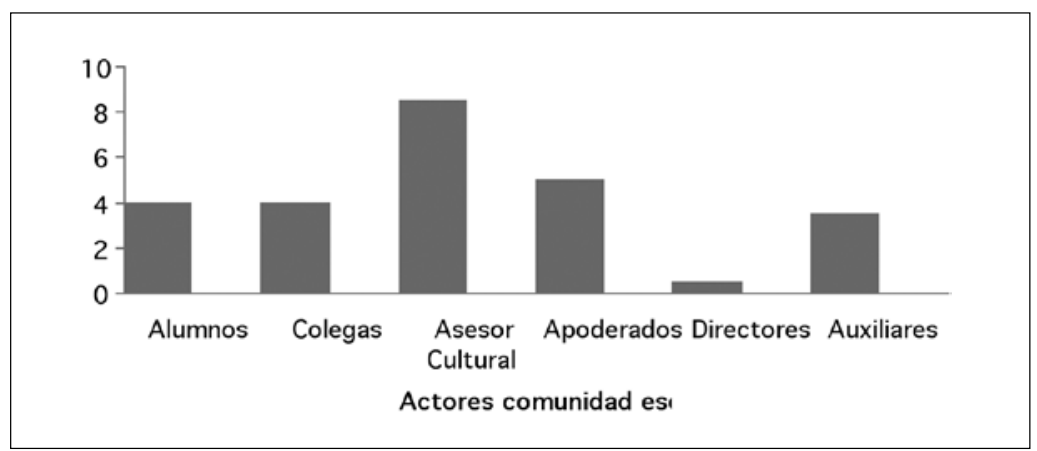

Puede observarse que, en la comunidad escolar, es el Asesor Cultural quien hace mayor uso de la lengua mapuche. Esto se entiende pues es él quien cumple la función de enseñar la lengua y la cultura a los escolares. Los niños prácticamente no hablan la lengua, al igual que los directores y coordinadores. Estas cifras son coherentes con la información anterior sobre el uso del mapuche en la comunidad y muestran, además, la incipiente presencia de la lengua vernácula en la escuela. Se debe reconocer que el castellano es la lengua de uso mayoritario en la escuela. Todas las clases se realizan en esta lengua y el mapudungun queda relegado a las dos o tres horas de clases que realiza el Profesor Tradicional o Asesor Cultural. En la dinámica escolar interna resulta evidente la supremacía del castellano sobre la lengua mapuche. Los hechos a partir de los cuales se sostiene esta afirmación pueden encontrarse en la relación lingüístico-cultural asimétrica que existe entre el vernáculo, propio de una etnia minoritaria, y la lengua de la sociedad y la adecuación curricular propia del estado nacional.

Sin embargo, se destaca en este aspecto el avance y reconocimiento que ha logrado la lengua y cultura mapuche en el ámbito escolar. Se dice esto a partir de consideraciones que históricamente han estigmatizado y marginado lo mapuche de los espacios educativos formales. En la actualidad, el mapudungun y lo mapuche se han incorporado en el currículum educativo y esto permite destacar el avance y la dignificación social de lo mapuche en la escuela.

El grado de dominio de la lengua mapuche desarrollado por los estudiantes, según se puede observar en el gráfico 3, se correlaciona con los datos del gráfico 1 , en el cual se muestra que tanto los niños como los jóvenes hablan con poca frecuencia. La proyección en este ámbito es negativa, toda vez que en la conservación y uso de la lengua los niños juegan un papel fundamental.

109 
Actitudes lingüísticas de profesores mapuche de Educación Básica: vigencia y enseñanza del mapudungun en el contexto educativo / Aldo Olate Vinet y Marisol Henríquez Barahona

Gráfico 3. Grado de dominio de la lengua mapuche por parte de los estudiantes

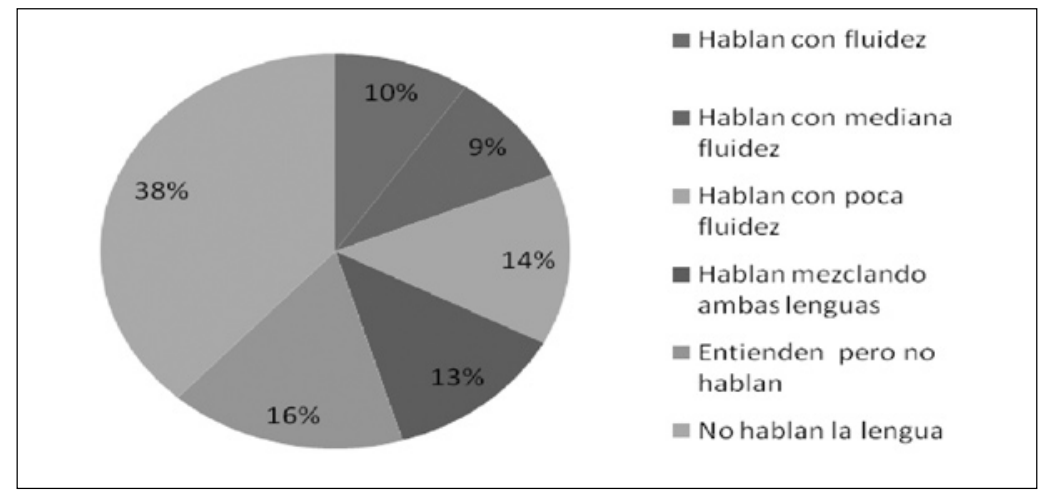

Frente a la pregunta ¿Con quiénes hablan mapudungun los niños en la escuela? (ver gráfico 4), las respuestas nos dicen que los padres y apoderados, al igual que los profesores no son muy activos en mapudungun en la escuela. Esto puede interpretarse por el hecho de que la escuela sigue siendo, para los apoderados, un ámbito de contacto formal con la sociedad occidental, la lengua de uso, por lo tanto, debe ser el castellano, lengua representativa de la tradición escolar en la comunidad. Los apoderados pueden ser hablantes del vernáculo, pero cuando están en la escuela con sus hijos prefieren el uso del castellano.

El Asesor Cultural, en cambio, cumple una tarea fundamental en la escuela, toda vez que se constituye como el encargado de la transmisión de la lengua y la cultura mapuche al interior de la comunidad escolar, ámbito en el cual el uso del mapudungun hasta hace pocos años era prohibido.

Gráfico 4. Con quiénes hablan lengua mapuche los niños en la escuela

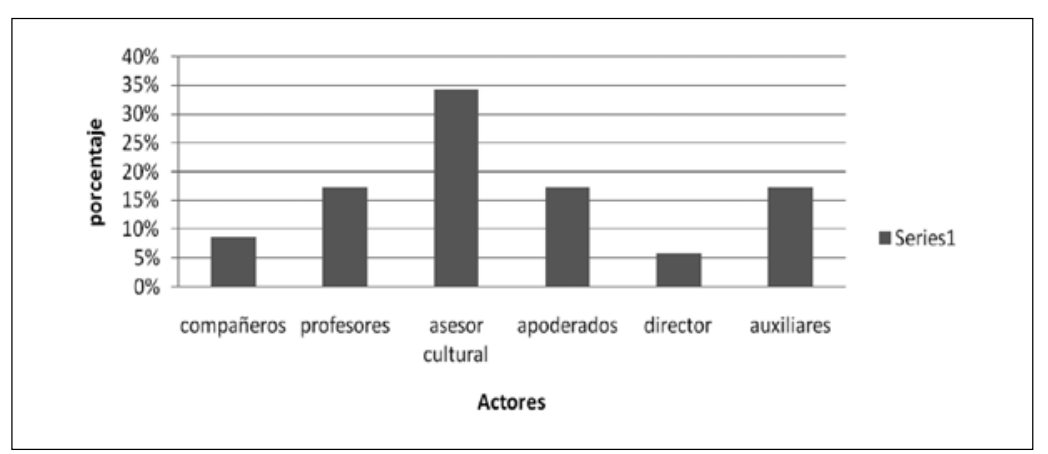

\subsection{Actitudes lingüísticas: Lealtad y Utilidad}

En este apartado se abordarán, en términos generales, las actitudes lingüísticas que los docentes presentan hacia el mapudungun y el castellano. Revisaremos las dimensiones de lealtad hacia la lengua (orgullo, fidelidad) y utilidad. En 
cuanto al primer concepto, se entenderá por lealtad tanto el orgullo que los hablantes demuestran en relación con el uso del vernáculo en diferentes ámbitos y el grado de entusiasmo hacia la lengua, como el grado fidelidad proyectado a través de la resistencia por parte de los hablantes a la pérdida de usos o a los cambios en la estructura de la lengua autóctona. (Blas Arroyo, 1994).

Por otro lado, la utilidad se entiende como la necesidad que existe de una lengua para la comunicación social en una determinada comunidad de habla (Blas Arroyo, 1994). En este sentido, nos interesa saber la utilidad que conceden los profesores a la lengua mapuche en el ámbito social y educativo.

El análisis que se propone es de naturaleza cualitativa debido a que interpreta una serie de reflexiones (Muñoz Cruz, 2006) a través de las cuales los docentes afirman tanto su identidad/lealtad con la lengua, como la utilidad de ésta.

En el dominio de la lealtad hacia la lengua los docentes muestran una actitud positiva. Todos los profesores afirman que están de acuerdo con la enseñanza del mapudungun en la escuela. Las interpretaciones que pueden realizarse demuestran un alto grado de compromiso con la lengua. Estas actitudes son de fidelidad lingüística debido a que los docentes sienten un compromiso con la difusión de la lengua como herramienta que permite la conservación de la cultura.

Las preguntas sobre el futuro de la lengua giraron en torno a tres alternativas: (1) se proponía que la lengua permanecería activa en el tiempo, (2) que se perdería progresivamente y (3) que desaparecería. Dentro de estas alternativas los docentes respondieron asumiendo una actitud crítica que demuestra un orgullo hacia la lengua.

Se dice lo anterior debido a que la gran mayoría propuso que la lengua permanecerá activa en el tiempo y que la escuela es un elemento fundamental para su conservación. Los argumentos que avalan dicho planteo son de varios tipos, desde una adscripción étnica íntegra: "siempre va a existir un mapuche que va a practicar su cultura, tradición y lengua materna" hasta críticas sobre la función del estado en este tema: "no hay una política educacional que pueda fortalecer la lengua"; "porque no existe una politica de gobierno".

Siguiendo en el análisis sobre la lealtad lingüística, se revisaron las respuestas de los docentes en lo referido a qué lengua debe aprenderse en la escuela. Nuevamente, el cuestionario presentó alternativas: (1) el castellano primero, (2) el mapudungun primero, (3) castellano y mapudungun al mismo tiempo y (4) sólo castellano. Las respuestas fueron de diversa naturaleza y los argumentos o razones bajo las cuales justificaron sus alternativas se centran principalmente en que si el niño aprende ambas lenguas al mismo tiempo "adquiere un mejor dominio lingüístico"; "siendo mapuche su autoestima se eleva y su identidad, y serían bilingües". Para los profesores lo ideal sería que ambas lenguas se enseñaran al mismo tiempo, esto lo ven como una ventaja tanto a nivel cognitivo como a nivel cultural. Estas respuestas deben entenderse como una actitud positiva de lealtad hacia la lengua vernácula. 
Actitudes lingüísticas de profesores mapuche de Educación Básica: vigencia y enseñanza del mapudungun en el contexto educativo / Aldo Olate Vinet y Marisol Henríquez Barahona

Con relación a la función social e importancia del mapudungun nos interesa conocer el grado de utilidad que los profesores conceden al mapuche. Para ello investigamos la opinión de éstos acerca de la necesidad de que sus estudiantes aprendan el vernáculo y qué motivos entregan.

Frente a la pregunta: ¿Es útil hablar mapudungun en estos tiempos? La totalidad de los docentes consultados respondió positivamente. Las razones esgrimidas se basan en las siguientes percepciones: "los niños mapuche tendrían su autoestima bien elevadas"; "se conserva la lengua de los ancestros"; "serviría para mucho, comunicación secreta, conocimientos ancestrales"; "para que no se pierda la lengua y perdure con el tiempo". Subyacen a dichas afirmaciones los valores que los actores generan en torno a la lengua. La utilidad en este caso no se dirige hacia una función social de la lengua, en términos de dominios o ámbitos de uso, sino hacia la valoración de ésta como un instrumento que permite la comunicación ancestral y que tiene una utilidad intraétnica.

Con respecto a la utilidad del castellano, los profesores igualmente respondieron positivamente, los juicios que se desprenden se vinculan con ideas que sostienen que el castellano es la lengua "con la que los niños se pueden comunicar de mejor forma con la otra sociedad", "porque es la lengua oficial del país", "no existen problemas para la realización de trámites en las ciudades", "cuando se vayan a trabajar o estudiar fuera solo se habla esta lengua, pero nunca deben olvidar su lengua mapuche". Se desprenden de dichas respuestas la importancia del castellano como lengua de desarrollo y de comunicación intercultural.

Ambas lenguas presentan utilidades diversas, por una parte la funcionalidad del mapudungun se vincula con la capacidad de conservar el conocimiento ancestral del pueblo y para la comunicación intraétnica, entendida como los circuitos comunicativos exclusivos donde se restringe el uso del castellano. El castellano, por otra parte, presenta rasgos de alta instrumentalización en cuanto a herramienta que permite el diálogo intercultural y desarrollo y progreso socioeconómico de los mapuche al insertarse en la sociedad occidental.

\subsection{Actitud docente hacia el PEIB}

También incluimos un apartado en el cuestionario para indagar en las actitudes de los profesores hacia el Programa de Educación Intercultural Bilingüe del que han sido o son parte actualmente. Creemos que es importante conocer la evaluación que los profesores hacen de este Programa a diez años de su implantación, aproximadamente, en las áreas mapuches, y relevar las fortalezas y debilidades que presenta desde la perspectiva de estos actores.

\subsubsection{Grado de acuerdo con el PEIB y su grado de efectividad}

Frente a la pregunta: ¿Está de acuerdo con la existencia del PEIB?, todos los docentes respondieron que están plenamente de acuerdo con la existencia de este Programa. Sin embargo, al preguntárseles por la efectividad de éste, la

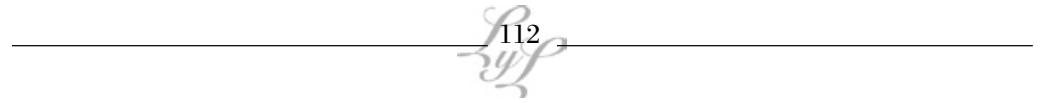


respuesta mayoritaria fue que no es del todo efectivo. Esto lo observamos en el gráfico 5:

Gráfico 5. Grado de efectividad del PEIB

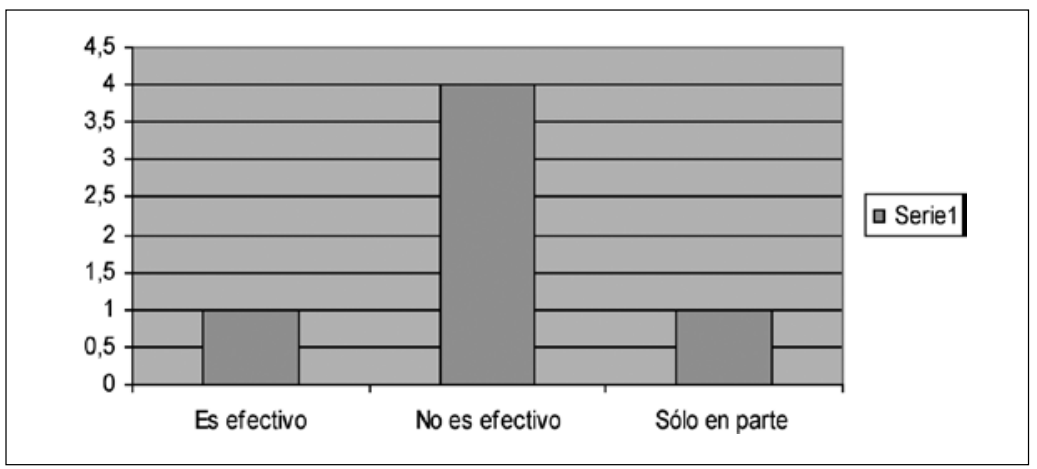

Las razones que justificarían la falta de efectividad del Programa, según los docentes, son de naturaleza variada, entre las cuales destacan:

- "La mayoría de estos Programas funcionan por la voluntad de los docentes y luego de la comunidad, si los docentes no incentivan, no son efectivos"

- "Para que funcionen se requiere de supervisión de especialistas en terreno"

- "El Ministerio de Educación no lo toma en serio"

- "Los Programas se terminan y no se pueden continuar por falta de recursos"

- "No se toma de raíz el tema"

\subsubsection{Autopercepción de su capacitación y evaluación de los pares}

Otra dimensión que se indagó se relaciona con la competencia de los docentes para trabajar en este Programa. En este ámbito, la mayoría de ellos declaró que se sentían capacitados y muy interesados en la cultura mapuche y sus problemáticas. Argumentaron tener voluntad para trabajar en el tema y poseer las herramientas académicas para hacerlo. Además, declararon que por el hecho de ser mapuche y hablantes tenían cierto conocimiento de la cultura y sensibilidad para abordar la educación intercultural. La pregunta que nos surgió entonces y que queda por responder es: ¿Qué opinión tendrán los docentes no mapuche y no hablantes sobre el PEIB?; ¿Es suficiente ser mapuche hablante para trabajar eficientemente en un programa de esta naturaleza?

Al evaluar la preparación de sus colegas para trabajar en este contexto, los entrevistados señalaron, que, si bien es cierto, existen colegas que están capacitados para trabajar en el Programa, otros, en cambio, no lo están o están 
Actitudes lingüísticas de profesores mapuche de Educación Básica: vigencia y enseñanza del mapudungun en el contexto educativo / Aldo Olate Vinet y Marisol Henríquez Barahona

medianamente capacitados. Entre las razones explicitadas se encuentran las siguientes:

- No están bien preparados porque no hay perfeccionamiento permanente hacia los docentes que ya están en el sistema y a los nuevos que ingresan cada año.

- No se interesan por aprender de la otra cultura.

\subsubsection{Evaluación general del PEIB}

En términos generales, los docentes consideran que este Programa beneficia a la comunidad mapuche y, en especial, a los escolares, por razones, tales como:

- Se consideran los contenidos y el conocimiento mapuche (kimün) en la escuela, lo que antiguamente no ocurría.

- Los niños salen fortalecidos en su autoestima.

- Los niños valoran más su identidad y cultura.

- El Asesor Cultural o Educador Tradicional es un aporte significativo, ya que entrega la visión y el conocimiento mapuche a los niños y profesores.

- El Asesor Cultural ayuda a los niños a fortalecer la lengua vernácula y costumbre de sus ancestros.

Con respecto a las debilidades, los encuestados señalaron sus aprehensiones principalmente frente al tratamiento del mapudungun, lo que no les parece adecuado, porque:

- Tiene pocas horas destinadas para ello.

- A veces está dentro del curriculum, pero generalmente es un electivo o taller.

- Los educadores tradicionales saben la lengua mapuche, pero no tienen las herramientas pedagógicas para enseñarlo.

- Se ve sólo superficialmente "como una pincelada".

Otra debilidad que destacan los docentes es la falta de textos para la enseñanza de la lengua y cultura mapuche y, como ya se dijo, la principal debilidad sería la falta de perfeccionamiento docente sistemático en el ámbito de la Educación Intercultural Bilingüe.

\section{Conclusiones}

Las primeras reflexiones que pueden proyectarse del presente estudio se vinculan con dos ideas puntuales, las cuales deben seguir profundizándose.

En primer lugar, sostenemos que el mapudungun, en las comunidades estudiadas, es una lengua vigente, hablada fundamentalmente por ancianos y adultos. En el ámbito escolar, en cambio, su uso es menor y es liderado prin- 
cipalmente por el Profesor Tradicional o Asesor Cultural. Algunos docentes y escolares también lo practican, pero en menor grado. El uso poco frecuente del mapudungun al interior de los establecimientos obedece a que la escuela cumple un papel protagónico en cuanto a la transmisión de códigos formales y escritos que detentan el prestigio social. Se ha documentado, en algunos estudios, la resistencia de grupos étnicos al uso de la lengua materna en la escuela (Pellicer y Rockwell, 2003). Sin embargo, a pesar de la importancia social que tiene el castellano, la lengua mapuche está tomandose espacios dentro de los ámbitos escolares. En este marco reflexivo general surgen dos cuestiones que deben investigarse. Por una parte, es necesario estudiar el grado de apropiación del castellano en las comunidades escolares que se desarrollan en ambientes interétnicos. Por otra, estudiar la importancia otorgada a la escritura entre las comunidades indígenas. En otras palabras, debemos analizar las actitudes hacia la oralidad y literacidad en contextos formales e informales. Con estos datos se pueden proyectar programas de apropiación curricular adecuados para las necesidades de la Educación en contextos interculturales.

En segundo lugar, puede observarse la existencia de criterios homogéneos entre los docentes entrevistados con respecto a las actitudes que tienen hacia la lengua mapuche, su importancia, valoración y proyección. Estas actitudes son positivas y favorecerían la conservación y difusión de la lengua y cultura mapuche en el contexto formal propio de la educación nacional. Dicha homogeneidad es relativa, debido a que la muestra escogida es pequeña. Por lo mismo, es menester ampliar el número de entrevistados para configurar una visión más global de la situación. Los docentes reconocen la importancia de la introducción del conocimiento y lengua mapuche en la escuela. Esto ha traído consecuencias positivas en la comunidad escolar, principalmente para los escolares, quienes reconocen su identidad y se acercan a su cultura.

La configuración de una muestra más representativa es imprescindible para confirmar el arribo definitivo de la lengua mapuche a los espacios escolares. Las actitudes previas que muestran los docentes son de naturaleza positiva y fomentan la identidad étnica y lingüística hacia lo mapuche, pero además, los profesores son concientes de la importancia del castellano, su escritura y uso público formal. Este contexto resultaría propicio para iniciar actividades de revitalización lingüística.

\section{Bibliografía}

Blas Arroyo, J. (1994). "Actitudes lingüísticas en la sociedad valenciana". En Hispania, 1 (77), 143-155.

Croese, R. (1983). "Algunos resultados de un trabajo de campo sobre las actitudes de los mapuches frente a su lengua materna". En RLA, 21, 23-34.

Fasold, R. (1996). La Sociolingüística de la sociedad. Introducción a la sociolingüística. Madrid: Visor libros, S.L. 
Actitudes lingüísticas de profesores mapuche de Educación Básica: vigencia y enseñanza del mapudungun en el contexto educativo / Aldo Olate Vinet y Marisol Henríquez Barahona

Fasold, R. (1998). Sociolingüística del lenguaje. Buenos Aires: DOCENCIA.

García Marcos, F. (1993). Nociones de sociolingüística. Barcelona: Ediciones Octaedro.

Gundermann, H., Canihuan, J., Clavería, J. y Faúndez, C. (2008). Perfil Sociolingüístico de Comunidades mapuche de la Región del Biobio, Araucanía, los Ríos y los Lagos. Informe de investigación. Santiago: CONADIUTEM.

Gundermann, H., Canihuan, J., Clavería, J. y Faúndez, C. (2009). "Permanencia y desplazamiento, hipótesis acerca de la vitalidad del mapuzugun". En RLA, 1 (47), 37-60.

Fernández, I. y Hernández, A. (1984) "Estudio exploratorio de actitudes en una situación de bilingüismo. El caso mapuche". En RLA 22, 35-51.

López Morales, H. (1993). Sociolingüística. Madrid : Editorial Gredos.

Ministerio de Educación [en línea]. http://www.mineduc.cl/index0.php?id_portal=28.

Moreno Fernández, F. (2005). Principios de sociolingüística y sociología del lenguaje. Madrid: Ariel.

Muñoz Cruz, H. (2006). "Cambio sociolingüístico y experiencias interculturales desde discursos autobiográficos de hablantes de lenguas indígenas". En cuadernos culturales, 4 (7), 23-48.

Pellicer, D. y Rockwell, E. (2003). "iAtajámelo ese llama! La apropiación del castellano en las escuelas bilingües de Puno". En Ingrid Jung y Juan Enrique López (Eds) Abriendo la escuela. Lingüistica aplicada a la enseñanza de lenguas. Madrid: Morata Ediciones.

Silva-Corvalán, C. (2001). Sociolingüística y Pragmática del español. Washington, D.C: Georgetown University Press.

Saville Troike, M. (2005). Etnografía de la comunicación. Buenos Aires: Prometeo libros.

Vergara, J. y Gundermann, H. (Eds). (2005). Descripción del contexto sociolingüístico en comunidades indigenas de Chile, MINEDUC Programa EIB Orígenes, Santiago de Chile. 